\title{
Difficulties in Diagnosis of Psittacosis or Ornithosis: A Case Report
}

\author{
Masoud Ebrahimi ${ }^{1}$, Abbas Fadii ${ }^{2}$, Masoud Mardani ${ }^{1}$, Kouros Aghazade ${ }^{3,4}$, Farshad Divsalar $^{5}$ and \\ Javad Minooeifar $^{6, *}$ \\ ${ }^{1}$ Infectious Diseases and Tropical Medicine Research Center, Shahid Beheshti University of Medical Sciences, Tehran, Iran \\ ${ }^{2}$ Labbafinejad Hospital, Shahid Beheshti University of Medical Sciences, Tehran, Iran \\ ${ }^{3}$ Department of Infectious Disease, Faculty of Medicine. AJA University of Medical Science, Tehran, Iran \\ ${ }^{4}$ Infectious Disease Research Center, AJA University of Medical Science, Tehran, Iran \\ ${ }^{5}$ Department of Infectious Disease and Tropical Medicine, Iran University of Medical Science, Tehran, Iran \\ ${ }^{6}$ Janbazan Medical and Engineering Research Center, Tehran, Iran \\ "Corresponding author: Janbazan Medical and Engineering Research Center, Farrokh St., Moghaddas Ardabili St., Shahid Chamran Highway, Tehran, Iran. Tel: +98-9121193741, \\ Fax: +98-2122418180, Email: jminooeefar@yahoo.com
}

Received 2018 January 01; Revised 2018 August 30; Accepted 2018 September 29.

\section{Abstract}

Introduction: Chlamydia psittaci is an intracellular and zoonotic pathogen that is transmitted through contact with birds. This pathogen causes various clinical manifestations, including subclinical cases and fatal cases. On the other hand, the variety and low specificity of many of the symptoms make diagnosis difficult while early detection of the disease causes a significant reduction in mortality.

Case Presentation: The patient was a 47-year-old man with a history of headache, fever, chills, dyspnea, and productive cough with a primary diagnosis of sinusitis, which was treated with co-amoxiclav. Due to the lack of recovery, the patient was hospitalized with the diagnosis of pneumonia and was treated with meropenem and levofloxacin. After showing no improvement and finding out about the history of contact with a parrot, the treatment was changed to Doxycycline and Ceftriaxone, which led to the reduction of symptoms. Eventually, due to the patient's positive serological tests, the diagnosis of Chlamydia Pneumonia was established and the patient was discharged with oral doxycycline.

Conclusions: As the fatality rate of psittacosis in absence of treatment is high, it is necessary to treat the patient immediately.

Keywords: Psittacosis, Birds, Doxycycline, CAP

\section{Introduction}

Chlamydia psittaci is an intracellular pathogen (1). It is a zoonotic pathogen that infects a wide range of birds as hosts and may cause severe disease when is transmitted to humans (2). Infected birds are often asymptomatic, but sometimes they may have symptoms like chills, anorexia, and diarrhea (3). Contaminated birds pass pathogens through their stool and nasal secretions, and humans get sick from contact with them (4). The disease caused by Chlamydia psittaci is called psittacosis or parrot fever (4). Since this disease is not restricted to parrot and other birds can also transmit microorganism, it is also called Ornithosis (3). Infection in humans mostly presents as communityacquired pneumonia (CAP), which is responsible for $1 \%$ $1.2 \%$ of all causes of $\operatorname{CAP}(5,6)$. Human-to-human transmission is rare and isolation and chemoprophylaxis are not necessary. This patient was chosen for the case presentation because of receiving various treatments. Diagnosis in the early stages of the disease and initiation of prompt treatment can reduce mortality from $20 \%$ to $1 \%$ (3).

\section{Case Presentation}

The patient was a 47-year-old man complaining of fever and chills, dyspnea, and cough that began two weeks before admission. The patient visited a doctor in the outpatient service at the early stage of disease, who diagnosed acute sinusitis and prescribed co-amoxiclav and clarithromycin subsequently. After seeing no improvement, he once again returned to the clinic and was admitted this time.

The patient or his family did not have a history of lung disease or other illnesses. He traveled to India in the last year. History revealed the presence of a parrot at home with no history of smoking. In his career, he had not been in contact with chemicals. The patient mentioned a weight loss of $6 \mathrm{~kg}$ in the last 2 weeks. The vital signs were: Temp. $38^{\circ} \mathrm{C}$, B / P 100/70 mm/Hg, RR 24 / Min and PR 90/Min. Clinical examination was normal except for bilateral coarse 
crackle in the lung. The examination of the heart did not have an abnormal point. The patient was admitted with a diagnosis of pneumonia and the treatment was started with meropenem and levofloxacin.

Laboratory investigation revealed leukocytosis with 16300 WBC and 78\% Polymorphonuclear (PMN) and platelet count was 453,000 and eosinophil and band-bone were higher than normal but other $\mathrm{CBC}$ indices were normal (Table 1). Collagen-vascular tests, including P-ANCA, C-ANCA, ANA, and Anti-dsDNA were reported normal. In the liver tests, alkaline phosphatase showed higher than normal range but other indices were normal. The CRP and ESR were 47 and 72, respectively.

In bronchoscopy, there was copious purulent discharge but other findings were normal. Bronchoalveolar lavage (BAL) was performed and the samples were sent to the laboratory for bacterial, mycobacterial and fungal smear and culture, which gram-positive cocci (Staphylococcus) and gram-negative bacilli were reported.

Due to the lack of recovery, the history of contact with a parrot and the previous antibiotic regimen (meropenem and levofloxacin), the treatment was changed to Doxycycline and Ceftriaxone. In the next visit, the fever discontinued and RR reached 16/Min. In subsequent tests, the patient's leukocyte was reduced to 10,200 with a predominance of $71 \%$ PMN. Serological test results for Chlamydia psittaci were IgM 1.5 RU/ML (normal range less than 0.8 $\mathrm{RU} / \mathrm{ML}$ ) and IgG $61.5 \mathrm{RU} / \mathrm{ML}$ (normal range less than 16 $\mathrm{RU} / \mathrm{ML}$ ), which were positive and diagnosis of pneumonia due to Chlamydia psittaci was established for the patient. Eventually, the patient was discharged in a good general condition with oral doxycycline.

\section{Discussion}

After 5 to 15 days of incubation period, the disease may start insidiously and suddenly lead to multiple syndromes. The disease may present as atypical pneumonia with nonproductive cough, fever, headache, and abnormal changes in chest graph, which is usually more than expected on the basis of clinical symptoms and examination (3). In a study, $11 \%$ of patients with probable or confirmed psittacosis had productive cough (7).

According to the signs and symptoms of the patient, he was hospitalized with the diagnosis of pneumonia, which was treated with meropenem and levofloxacin. Finally, due to no relief in symptoms, history of contact with a parrot, and the possibility of chlamydial induced pneumonia psittaci, antibiotics were changed to Doxycycline and Ceftriaxone. The patient responded quickly to the treatment. It is notable that in one study, $60 \%$ of patients with probable or definite psittacosis had no history of contact with birds (7). There are many differential diagnoses that are variable according to psittacosis-induced syndromes, for example in the atypical pneumonia form, viral pneumonia, Q fever, Legionellosis, Mycoplasmal pneumonia are considered to be differential diagnoses (3). Although pneumonia is the most common manifestation of psittacosis, all organs can be involved in this disease (8). Several cases of severe pneumonia with ARDS and pericarditis have been reported (9). Severe pneumonia may be the primary manifestation of the disease that led to ICU admission (10).

In a study of 85 patients with suspected Psittacosis, 48 cases were confirmed as definitive or probable psittacosis (7). In another study that reviewed 11 articles over a 30-yearperiod, it was found that in patients with psittacosis and severe respiratory insufficiency, 10 out of 12 patients had exposure to birds as a major risk factor. Severe hypoxemia and kidney failure were associated with poor prognosis. Eight patients died of psittacosis or complications of the infection (11).

The most common symptom is fever, occurring in 50 to $100 \%$ of cases but most often in the last stages of the disease. Headache and myalgia have been reported in 30 to 70 percent of the cases; however, these symptoms are misleading until the cough occurs due to lack of specificity. Even at this time, there are many distinct diagnoses. The most common findings in the examination are fever, throat erythema, rale or other abnormal findings in lung auscultation and hepatosplenomegaly. Horder's Spot, one of the cutaneous manifestations of psittacosis, is a maculopapular lesion on the chest or trunk that is pink. The involvement of other organs, such as cardiac and kidney involvement, hepatitis, neurological manifestations, etc., are consequences of the systemic nature of the disease (3). In one study, the prevalent symptoms were fever, cough, headache, myalgia, nausea, diarrhea, chills, sputum, chest pain and shortness of breath (7). Our patient's presentations were pulmonary involvement, headache, fever, chill, and productive cough.

In the $\mathrm{CBC}$ test, WBC usually is normal or increased slightly and two-thirds of cases have a leftward shift. In 50\% of cases, liver tests are abnormal. The CXR is abnormal in $75 \%$ of cases, which is more than expected on the basis of respiratory signs and symptoms. The most common abnormal appearance in X-ray is a consolidation in a single lower lobe. Other patterns include ground glass opacity (GGO), patchy view, segmental view, or lobar and miliary view, and sometimes have Halo Sign view that may be misleading with other respiratory illnesses. In 50\% of cases, we have pleural effusion, which is small and asymptomatic (3). In this patient, leukocytosis was observed with the leftward shift and patchy infiltration in right lower lobe in $\mathrm{X}$ ray (Figure 1). 


\begin{tabular}{|c|c|c|c|c|}
\hline Test & First Day & Third Day & Normal Range & Flag \\
\hline WBC & 16300 & 10200 & $4500-11000$ & High \\
\hline Neutrophil, \% & 77.9 & 71.5 & $55-75$ & High \\
\hline Lymphocyte, \% & 12.4 & 18.1 & $20-45$ & Low \\
\hline ESR 1st hour, $\mathbf{m m} / \mathbf{h}$ & 72 & - & $0-22$ & High \\
\hline Chlamydia psittaci (IgM), RU/mL & 1.5 & - & $<0.8$ & High \\
\hline Chlamydia psittaci (IgG), RU/mL & 61.5 & - & $<16$ & High \\
\hline
\end{tabular}

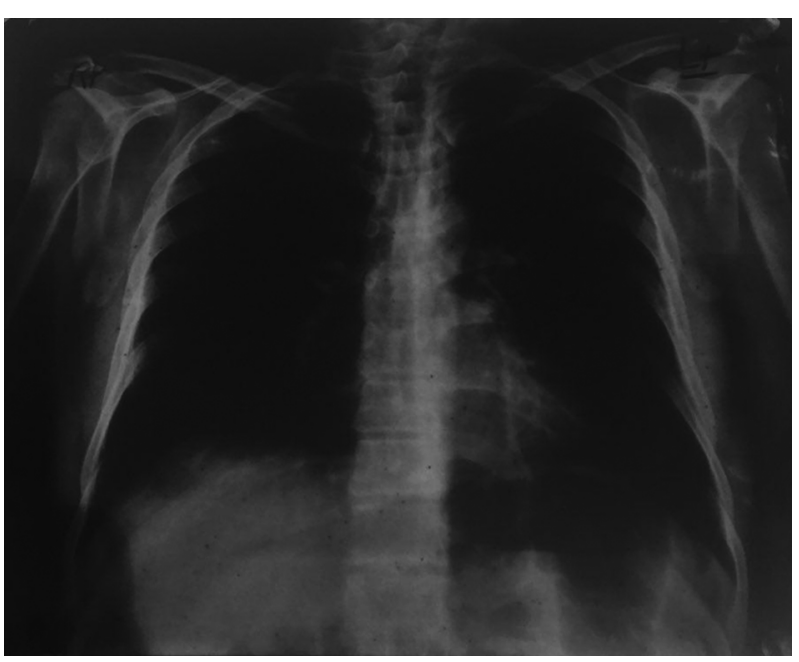

Figure 1. There is a patchy infiltration in RLL in the lung graphy affecting the right diaphragm border

According to the CDC, the definitive case of psittacosis is a person with consistent clinical symptoms and laboratory confirmation having one of the following criteria:

1-A titer of 1/16 IgM by MIF (microimmunofluorescence) method.

2- Positive culture of respiratory secretions.

3- Four-fold or greater rise in titer in CF (Complement fixation) or MIF antibody to a titer $1 / 32$ in the specimens with two weeks intervals.

The probable case is an individual with compatible symptoms along with a confirmed history of contact with a patient with confirmed disease or a titer of $1 / 32$ in a sample by CF or MIF assay (3). Based on a single serologic test, which was positive, the patient is considered a probable case of psittacosis.

The blood and sputum cultures are possible in the first 4 days and the first two weeks of the disease, respectively but usually cultures are not considered. Because the risk for laboratory staff and serological method is preferred (3).
In a study, because of the cross-reactions of old methods such as cultural, serological, and MIF assays, the replacement of new molecular methods such as PCR and RT-PCR for diagnosis has been discussed and was emphasized that modern methods are more standardized (12).

Drug of choice is doxycycline, $100 \mathrm{mg}$ twice daily or tetracycline $500 \mathrm{mg}$ four times a day for 10 to 21 days. Macrolides are effective in vitro and are recommended as alternative therapies in children and pregnant women; however, they may be less effective in severe cases and do not protect the fetus during pregnancy. Fluoroquinolones need more clinical experiences. Most patients feel better within 24 hours with therapy. Without treatment, the fatality rate of psittacosis is approximately $20 \%$; however, it is reduced to $1 \%$ following the treatment (3).

\subsection{Conclusions}

Given the high mortality rate of disease in the absence of treatment, it is necessary that the disease is quickly diagnosed and treated. Due to the non-specificity of the symptoms and on the other hand, the absence of contact with birds in a considerable number of patients, many differential diagnoses may be possible and achieving an accurate diagnosis is more difficult. However, paying attention to the symptoms and history of contact with birds can improve the diagnosis. As soon as the disease is diagnosed, the treatment should be started and continued for 10 to 21 days. The shorter course of the treatment will be associated with possible recurrence.

\section{Footnotes}

Authors' Contribution: Javad Minooeifar and Masoud Ebrahimi were responsible for the study design. Abbas Fadii, Masoud Mardani, Kouros Aghazade, Farshad Divsalar, Javad Minooeifar, and Masoud Ebrahimi analyzed and interpreted the data. Masoud Ebrahimi and Javad Minooeifar wrote the manuscript. All authors read and approved the final manuscript. 
Conflict of Interests: The authors declared they had no conflict of interests.

Ethical Approval: Our research protocol was submitted for consideration, comment, guidance, and approval to the Research Ethics Committee of Infectious Diseases and Tropical Medicine Research Center and accepted before the study begins.

Financial Disclosure: The authors have no financial interests related to the material in the manuscript.

Funding/Support: It is not declared by the authors.

Patient Consent: It is not declared by the authors.

\section{References}

1. Escalante-Ochoa C, Ducatelle R, Haesebrouck F. The intracellular life of Chlamydia psittaci: How do the bacteria interact with the host cell? FEMS Microbiol Rev. 1998;22(2):65-78. doi: 10.1111/j.15746976.1998.tb00361.x. [PubMed: 9729764].

2. Cong W, Huang SY, Zhang XX, Zhou DH, Xu MJ, Zhao Q, et al. Chlamydia psittaci exposure in pet birds. J Med Microbiol.2014;63(Pt 4):578-81. doi: 10.1099/jmm.0.070003-0. [PubMed: 24445511].

3. Bennett JE, Dolin R, Blaser MJ. Mandell, douglas, and bennett's principles and practice of infectious diseases E-book. Elsevier Health Sciences; 2014.

4. Balsamo G, Maxted AM, Midla JW, Murphy JM, Wohrle R, Edling TM, et al. Compendium of measures to control chlamydia psittaci infection among humans (psittacosis) and pet birds (avian chlamydiosis),
2017. J Avian Med Surg. 2017;31(3):262-82. doi: 10.1647/217-265. [PubMed: 28891690].

5. Hogerwerf L, D. E. Gier B , Baan B, Van Der Hoek W . Chlamydia psittaci (psittacosis) as a cause of community-acquired pneumonia: A systematic review and meta-analysis. Epidemiol Infect. 2017;145(15):3096105. doi: 10.1017/S0950268817002060. [PubMed: 28946931].

6. Spoorenberg SM, Bos WJ, van Hannen EJ, Dijkstra F, Heddema ER, van Velzen-Blad $\mathrm{H}$, et al. Chlamydia psittaci: A relevant cause of community-acquired pneumonia in two Dutch hospitals. Neth J Med. 2016;74(2):75-81. [PubMed: 26951352].

7. Branley JM, Weston KM, England J, Dwyer DE, Sorrell TC. Clinical features of endemic community-acquired psittacosis. New Microbes New Infect. 2014;2(1):7-12. doi: 10.1002/2052-2975.29. [PubMed: 25356332]. [PubMed Central: PMC4184616].

8. Stewardson AJ, Grayson ML. Psittacosis. Infect Dis Clin North Am 2010;24(1):7-25. doi: 10.1016/j.idc.2009.10.003. [PubMed: 20171542].

9. Haas LE, Tjan DH, Schouten MA, van Zanten AR. [Severe pneumonia from psittacosis in a bird-keeper]. Ned Tijdschr Geneeskd. 2006;150(3):117-21. Dutch. [PubMed: 16463610].

10. Wainwright AP, Beaumont AC, Kox WJ. Psittacosis: Diagnosis and management of severe pneumonia and multi organ failure. Intensive Care Med.1987;13(6):419-21. [PubMed: 3668076].

11. Verweij PE, Meis JFGM, Eijk R, Melchers WJG, Galama JMD. Severe human psittacosis requiring artificial ventilation: Case report and review. Clinical infectious diseases. 1995;20(2):440-2. doi: 10.1093/clinids/20.2.440

12. Rodriguez-Dominguez M, Sanbonmatsu S, Salinas J, Alonso R, Gutierrez J, Galan JC. [Microbiological diagnosis of infections due to Chlamydia spp. and related species]. Enferm Infecc Microbiol Clin. 2014;32(6):380-5. Spanish. doi: 10.1016/j.eimc.2013.01.015. [PubMed: 23523029]. 